\title{
Overexpression of ETV4 protein in triple-negative breast cancer is associated with a higher risk of distant metastasis
}

\author{
Zhong-Yu Yuan ${ }^{1-3, *}$ \\ Ting Dai ${ }^{1,2, *}$ \\ Shu-Sen Wang ${ }^{1-3}$ \\ Rou-Jun Pengl-3 \\ Xing-Hua $\mathrm{Li}^{1,2}$ \\ Tao Qin ${ }^{1-3}$ \\ Li-Bing Song ${ }^{1,2}$ \\ Xi Wang ${ }^{1,2,4}$
}

'State Key Laboratory of Oncology in South China, Guangzhou, People's Republic of China; ${ }^{2}$ Collaborative Innovation Center for Cancer Medicine, Guangzhou, People's Republic of China; ${ }^{3}$ Department of Medical Oncology, Sun Yat-Sen University Cancer Center, Guangzhou, People's Republic of China;

${ }^{4}$ Department of Breast Oncology, Sun Yat-Sen University Cancer

Center, Guangzhou, People's

Republic of China

*These authors contributed equally to this work

Correspondence: Zhong-Yu Yuan Department of Medical Oncology, Sun Yat-Sen University Cancer Center, 65I Dongfeng Road East,

Guangzhou 510060, People's Republic of China

Tel +862087343794

Fax +86 2087343535

Email yuanzhygz@।63.com

\section{XiWang}

Department of Breast Oncology, Sun Yat-Sen University Cancer Center, Guangzhou 5 I0060, People's Republic of China

Tel +86208734 3333

Fax +86 $2087343|7|$

Email wangxi@sysucc.org.cn
This article was published in the following Dove Press journal:

OncoTargets and Therapy

26 September 2014

Number of times this article has been viewed

Background: Patients with triple-negative breast cancer (TNBC) present a higher probability of distant metastasis and lack of effective targeted therapy. ETS translocation variant 4 (ETV4) is an ETS (E-26) transcription factor and has been associated with tumor metastasis. However, the clinical and functional significance of ETV4 in TNBC still remains unclear.

Methods: A human tumor metastasis polymerase chain reaction array was used to profile differential expression of tumor metastasis-related genes in TNBC tissue. Real-time reverse transcription and Western blot analyses were performed to verify ETV4 expression in TNBC cells and tissue. Immunohistochemistry was used to detect expression of ETV4 protein in 135 TNBC tissue samples for association between ETV4 protein expression and clinical outcomes. Results: A total total of eight upregulated (CCL7, KISS1, MET, MMP7, NR4A3, ETV4, TIMP3, and TSHR) and three downregulated (ITGA7, SSTR, and MMP2) genes were identified between TNBC tissue and the luminal subtype of breast cancer tissue. ETV4 messenger ribonucleic acid was more than five-fold upregulated in TNBC tissue compared with the control tissue. ETV4 overexpression was found in $57.0 \%$ of 135 TNBC cases. Overexpression of ETV4 protein was associated with an advanced stage and a higher proportion of positive lymph node and lymphovascular invasion. Patients with an ETV4-overexpressed tumor had a significantly higher risk of developing distant metastasis $(P<0.0001)$ and shorter overall survival and disease-free survival. Overexpression of ETV4 protein was an independent predictor of short disease-free survival of TNBC patients $(P=0.021)$.

Conclusion: Overexpression of ETV4 protein increases risk of developing distant metastasis and results in a poor prognosis for TNBC patients. Thus, ETV4 might be a novel target for developing an alternative therapeutic strategy for prevention of TNBC distant metastasis.

Keywords: breast carcinoma, triple-negative, ETS translocation variant 4, ETV4, prognosis

\section{Introduction}

Breast cancer is the most common malignancy among women in the world and accounts for $22.9 \%$ of all worldwide cancer cases and $>450,000$ cancer-related deaths annually. Due to the recent advancement in early detection, prevention, and treatment options, survival of breast cancer patients has significantly improved, especially for estrogen receptor (ER)- and progesterone receptor (PR)-positive breast cancer. However, there is less improvement for triple-negative breast cancer (TNBC). TNBC, a lack of ER, PR, and HER 2 expression, accounts for $15 \%-20 \%$ of all breast cancer cases. ${ }^{1-3}$ TNBC is characterized by poor survival and a high probability of early treatment relapse, especially distant metastasis. ${ }^{46}$ Due to the lack of hormone receptor and HER2 expression, treatment options for patients with TNBC are limited to cytotoxic chemotherapy. 
Thus, there is an urgent need for elucidation of novel targets for TNBC therapy and to improve the poor prognosis for the patients. The proclivity of breast cancer metastasis to particular organs may be mediated by specific genes. ${ }^{7-9}$

ETS translocation variant 4 (ETV4) is a member of the PEA3 subfamily of ETS (E-26) transcription factor. ${ }^{10}$ Due to sharing conserved ETS deoxyribonucleic acid (DNA) binding domains, ETV4 binds to targeting gene promoter dictated by specific sequence elements bearing a conserved 5'-GGAA/T-3' motif. ${ }^{11}$ ETV4 protein is generally expressed at low levels in normal adult tissue, but the expression levels of messenger ribonucleic acid (mRNA) and protein are much higher in several cancer tissue types, including breast, colorectal, and esophageal cancers and ovarian tumor, implicating the possibility of ETV4 as an oncogenic protein to promote cancer development and progression. ${ }^{12-16}$ The most frequently studied role of ETV4 in cancer development and progression is its ability to promote cancer metastasis. ${ }^{13,14,17}$ For example, a previous study showed that the nuclear translocation of ETV4 was induced by epidermal growth factor receptor and that the elevated ETV4 expression might promote ovarian tumor cell invasion by activating matrix metalloproteinase (MMP)-9 and MMP-14 expression. ${ }^{14}$ In breast cancer, ETV4 was reported to be overexpressed in estradiol receptor-negative breast cancer cell lines and in estradiol receptor-negative tissue. ${ }^{18-21}$ These data together indicate that ETV4 protein may play a role in regulation of tumor progression and distant metastasis.

Thus, in the current study, we first performed a tumor metastasis-related gene expression array analysis in TNBC tissue specimens and found that expression of ETV4 protein was induced; thus, we then detected expression of ETV4 protein in a larger sample size of TNBC tissue specimens for the association of ETV4 expression with clinicopathological features and disease relapse and prognosis of the patients. Our data demonstrated that overexpression of ETV4 protein was associated with increasing risk of TNBC distant metastasis and poor survival of the patients.

\section{Materials and methods \\ Breast cancer tissue samples}

In this present study, we obtained five cases of fresh TNBC tissue and five cases of luminal subtype fresh breast tumor tissue from the Department of Breast Surgery, Sun Yat-Sen University Cancer Center, Guangzhou, People's Republic of China. TNBC refers to ER negative, PR negative, and HER2 negative; luminal subtype refers to ER and/or PR positive but HER2 negative. With respect to ER or PR expression determined by immunohistochemistry (IHC), detection of $<1 \%$ neoplastic cells was considered negative. HER2 was determined initially by IHC and graded from 0 to $3+$. HER 2 negative was defined as HER 2 graded 0 or $1+$, and positive was defined as $3+$. Scores of HER2 2+ were confirmed as either HER2 negative or HER2 positive according to fluorescence in situ hybridization analysis.

In addition, to detect ETV4 expression, we also obtained 135 female patients with TNBC diagnosed without any evidence of distant metastasis at the time of surgery between January 2000 and December 2008 at Sun Yat-Sen University Cancer Center. The cases selected were based on distinctive pathologic diagnosis of invasive breast cancer, availability of complete medical records and follow-up data, but lack of ER, PR, and HER2 expression. All patients were staged according to the American Joint Committee on Cancer Tumor Node Metastasis Staging System for Breast Cancer (AJCC 2010, seventh edition). The formalin-fixed and paraffinembedded tissue specimens were retrieved from the archives of the Department of Pathology of Sun Yat-Sen University Cancer Center. Tissue microarrays (TMAs) were then constructed as described previously. ${ }^{22}$ Each sample was arrayed in triplicate to minimize tissue loss and to overcome tumor heterogeneity. In addition, normal breast tissue was obtained from three patients undergoing breast biopsies, who were subsequently not diagnosed with breast cancer.

All sample donors gave written informed consent. The use of these samples involved in our study was approved by the Institute Research Medical Ethics Committee of Sun Yat-Sen University.

\section{Tumor metastasis-related gene expression profiling}

Differential expression of tumor metastasis-related genes was analyzed in a Human Tumor Metastasis PCR (polymerase chain reaction) Array (APHS-028A; SuperArray Inc.) obtained from Kangchen Genechip (Shanghai, People's Republic of China) according to the manufacturer's instructions. Briefly, RNA was extracted from TNBC tissue using Trizol (Invitrogen, Carlsbad, CA, USA) and cleared up using an RNeasy ${ }^{\circledR}$ MinElute ${ }^{\mathrm{TM}}$ Cleanup Kit (Qiagen, Valencia, CA, USA). Subsequently, total RNA samples were reversely transcribed into complementary DNA (cDNA) using SuperScript III Reverse Transcriptase (Invitrogen), and these cDNA samples were amplified by PCR in $2 \times$ Super Array PCR Master Mix (SuperArray Bioscience, Frederick, MD, USA). Real-time PCR was then performed on each sample using the Human Tumor Metastasis RT $^{2}$ Profiler ${ }^{\mathrm{TM}}$ 
PCR Array (SuperArray Bioscience) in an Opticon ${ }^{\mathrm{TM}}$ DNA Engine ABI Prism system (Applied Biosystems, Foster City, CA, USA), according to the manufacturer's instructions. Data were normalized for glyceraldehyde 3-phosphate dehydrogenase (GAPDH) levels by the $\Delta \Delta \mathrm{Ct}$ method.

\section{Cell lines and culture}

Breast cancer T47D, MDA-MB-453, MDA-MB-415, MCF-7, MDA-MB-231, MDA-MB-435, ZR-75-30, and BT474 cell lines were obtained from ATCC and cultured with Dulbecco's Modified Eagle's Medium (Gibco BRL, Carlsbad, CA, USA) supplemented with 10\% fetal bovine serum (HyClone) in a humidified incubator with $5 \% \mathrm{CO}_{2}$ and $95 \%$ air.

\section{Protein extraction and Western blot}

Cultured cells at 70\%-80\% confluence were harvested, washed three times in phosphate-buffered saline, and lysed in a sodium dodecyl sulfate lysis buffer. Western blot analysis was performed using an anti-ETV4 antibody (Sigma-Aldrich, St Louis, MO, USA). The blotted membranes were then stripped and reprobed with anti-GAPDH as a loading control.

\section{RNA extraction and real-time reverse transcription PCR}

Total RNA was extracted from cultured cells using a Trizol reagent (Invitrogen) according to the manufacturer's instructions, and then $1.5 \mu \mathrm{g}$ of total RNA samples of each cell line was used for cDNA synthesis with random hexamers. The cDNA samples were subsequently amplified and quantified using the ABI Prism 7500 Sequence Detection System (Applied Biosystems) with SYBR Green I dye (Molecular Probes, Invitrogen) with ETV4 primers (5'-CCCATCTCAGTCACT GGCATT-3' and 5'-CCGCCAACATCTCCTTGbTTC-3') and GAPDH (5'-ACCACAGTCCATGCCATCAC-3' and 5'-TCCACCACCCTGTTGCTGTA-3'). The expression of ETV4 was normalized to the internal reference GAPDH levels using the formula 2-[(Ct of ETV4)-(Ct of GAPDH)], where $\mathrm{Ct}$ represents the threshold cycle for each transcript.

\section{IHC}

TMA blocks containing 135 cases of TNBC tissue samples were cut into $5 \mu \mathrm{m}$ sections and processed for IHC as described previously. ${ }^{22}$ After the TMA sections were incubated with a primary antibody against ETV4 (1:500; Santa Cruz Biotechnology, Santa Cruz, CA, USA) overnight at $4^{\circ} \mathrm{C}$, the stained TMA sections were reviewed and quantitatively analyzed using the AxioVision Rel. 4.6 computerized image analysis system assisted by an automatic measurement program (Carl Zeiss,
Oberkochen, Germany). Meanwhile, according to the manufacturer's recommendations, lymphocytes and K-562 cells were used as negative controls and positive controls, respectively. The staining intensity score (representing the average staining intensity of ETV4-positive cells) was assigned as 1, no staining; 2, weak staining; 3 , moderate staining; and 4, strong staining. Percentage of tumor cells stained was scored as $1,<10 \%$; $2,10 \%-30 \% ; 3,30 \%-75 \%$; and $4,>75 \%$. After that, these two scores were multiplied to calculate the staining index and obtain values of $1,2,3,4,6,8,9,12$, or 16 . A median staining index for all of the TNBC samples was used as a cutoff value to define high and low expression of ETV4, and then a score of $\geq 8$ was defined as high ETV4 expression and scores of $\leq 6$ defined as low ETV4 expression. All cases were scored by two independent observers.

\section{Statistical analysis}

Clinicopathological parameters were assessed between two subgroups by the chi-square test. Cumulative survival probabilities were calculated through the Kaplan-Meier method. Survival rates were compared by log-rank test. Multivariate analyses were performed by the Cox regression model. Subsequently, variables with traditional prognostic factors such as age at diagnosis, tumor size, lymph node involvement, pathologic stage, and lymphovascular invasion were included to the multivariate analysis with enter model. All statistical tests were two-tailed, and $P<0.05$ was considered significant All statistical analyses were performed using SPSS 17.0 (SPSS Inc., Chicago, IL, USA).

In addition, disease-free survival (DFS) was defined as the interval from the date of operation to the date of first recurrence. Overall survival (OS) was calculated as a period from the date of diagnosis to the date of death. Locoregional relapse-free survival was defined as the interval from the first treatment for breast cancer to the first locoregional relapse. Distant metastasis-free survival was calculated as a period from the date of diagnosis to the date of distant metastasis or contralateral breast cancer. Locoregional relapse was defined as the recurrence either in the treated breast or in the ipsilateral lymph node-bearing area (axillary, internal mammary, supraclavicular node).

\section{Results Upregulation of ETV4 expression in TNBC tissue specimens}

In this study, we first profiled differential expression of tumor metastasis-related genes in five cases of TNBC tissue samples compared with that of five luminal subtype 
(ER and/or PR positive and HER2 negative) breast tumor tissue using a Human Tumor Metastasis RT $^{2}$ Profiler ${ }^{\mathrm{TM}}$ PCR Array containing 84-cell metastasis-related genes. Our data showed that there are a total of eight upregulated (CCL7, KISS1, MET, MMP7, NR4A3, ETV4, TIMP3, and TSHR) and three downregulated (ITGA7, SSTR, and MMP2) genes (more than three-fold) identified in between TNBC tissue and luminal subtype breast cancer tissue. The levels of ETV4 mRNA in TNBC tissue were more than five-fold upregulated compared with those of luminal subtype breast tumor tissue (Table 1 and Figure 1), suggesting that the ETV4 gene might be involved in TNBC distant metastasis.

\section{Increased ETV4 expression in TNBC cell lines and tissue specimens}

To verify the array data, we performed Western blotting and real-time PCR analyses of ETV4 expression in TNBC cell lines and tissue specimens. We found that the expression of both ETV4 mRNA and protein was notably upregulated in hormone receptor-negative or TNBC cell lines tested (MDAMB453, MDA-MB-231, and MDA-MB-435) compared with hormone receptor-positive cells (T47D, MDA-MB-415, MCF-7, ZR-75-30, BT474; Figure 2A and B). Furthermore, the data on eight pairs of TNBC tissue and adjacent normal breast tissue samples showed that expression of ETV4 mRNA and protein was also significantly higher in TNBC tissue than in the adjacent normal breast tissue (Figure 2C-E), indicating that ETV4 is overexpressed in TNBC cells and tissue. Also, results of IHC on eight pairs of tissue samples as described

Table I Changes in relative expression for tumor metastasis genes between triple-negative breast cancer (TNBC) and luminal A breast cancer tissue

\begin{tabular}{lllll}
\hline Gene & $\begin{array}{l}\text { Fold change } \\
\text { TNBCI } \\
\text { luminal A }\end{array}$ & $\begin{array}{l}\boldsymbol{t} \text {-test } \\
\boldsymbol{P} \text {-value }\end{array}$ & $\begin{array}{l}\text { Average } \\
\text { raw Ct } \\
\text { TNBC }\end{array}$ & $\begin{array}{l}\text { Average } \\
\text { raw Ct } \\
\text { luminal A }\end{array}$ \\
\hline CCL7 & 3.06 & 0.0196 & 26.76 & 28.21 \\
ETV4 & 5.76 & 0.0033 & 24.78 & 28.39 \\
KISSI & 3.02 & 0.0329 & 27.98 & 29.40 \\
MET & 4.39 & 0.0184 & 23.51 & 25.48 \\
MMP7 & 6.09 & 0.0264 & 20.07 & 22.51 \\
NR4A3 & 3.20 & 0.0119 & 25.33 & 26.84 \\
TIMP3 & 4.39 & 0.0309 & 31.38 & 33.34 \\
TSHR & 4.48 & 0.0190 & 25.95 & 27.94 \\
ITGA7 & -8.25 & 0.0063 & 35.00 & 31.79 \\
MMP2 & -3.02 & 0.0101 & 20.13 & 18.37 \\
SSTR2 & -4.88 & 0.0234 & 27.00 & 24.55 \\
\hline
\end{tabular}

Notes: The table lists genes that exhibit at least a three-fold difference in expression in the TNBC tissue when compared with the luminal A breast cancer tissue. The raw threshold cycle $(\mathrm{Ct})$ values seen in the two samples are also listed for comparison.

Abbreviations: ETV4, ETS translocation variant 4; MMP, matrix metalloproteinase.

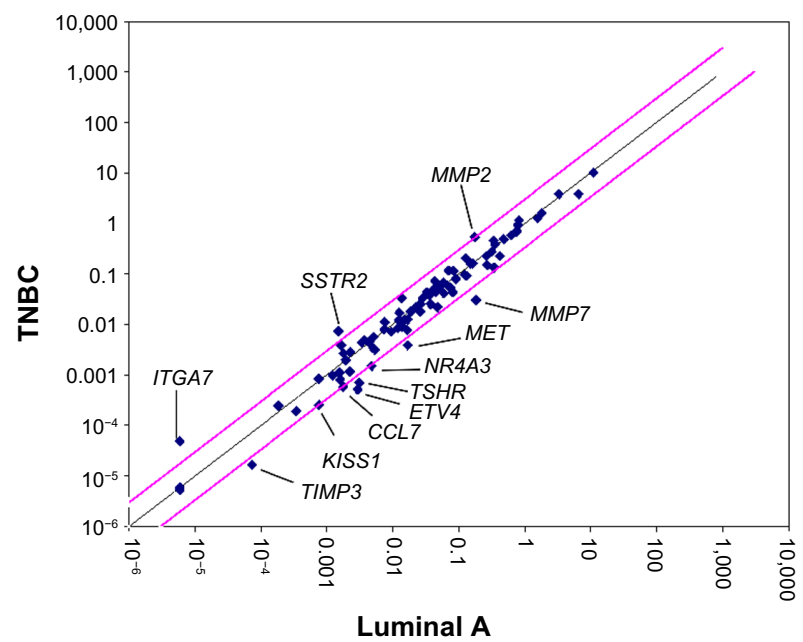

Figure I Relative expression comparison for 84 metastasis-related genes between triple-negative breast cancer (TNBC) and luminal A breast cancer tissue detected using a Human Tumor Metastasis $\mathrm{RT}^{2}$ Profiler ${ }^{\mathrm{TM}} \mathrm{PCR}$ Array.

Notes: The figure depicts a log transformation plot of the relative expression level of each gene $(\triangle \Delta C t)$ between TNBC ( $y$-axis) and luminal $A$ (x-axis). The red lines indicate a three-fold change in gene expression threshold.

Abbreviations: PCR, polymerase chain reaction; ETV4, ETS translocation variant 4; MMP, matrix metalloproteinase.

showed that ETV4 protein was positively expressed in TNBC tissue but not in the adjacent normal breast tissue.

\section{IHC analysis of ETV4 expression in TNBC tissue specimens}

To further explore whether ETV4 overexpression in TNBC was associated with the clinical outcomes, we analyzed ETV4 expression via IHC in 135 paraffin-embedded archived TNBC tissue samples. As shown in Figure 3, ETV4 staining was mainly localized to the nuclei of cancer cells. ETV4 staining was not detected in any of the normal breast tissue. In contrast, strong ETV4 staining was observed in the tumor cells of TNBC. Using the median staining score of ETV4 in TNBC as a cutoff value, 77/135 (57.0\%) cases displayed high levels of ETV4 expression and 58/135 (43.0\%) cases displayed low levels of ETV4 expression in TNBC tissue.

\section{Association of ETV4 expression with clinical outcome of TNBC patients}

We then associated ETV4 expression with clinicopathological parameters from these 135 patients. The association between ETV4 protein expression and various clinicopathological parameters is listed in Table 2. Specifically, compared with tumors with low ETV4 protein expression, patients with ETV4 highly expressed tumors have advanced AJCC stage and a higher proportion of positive lymph node and lymphovascular invasion (Table 2).

Next, we associated ETV4 expression with recurrence and survival of the patients. Up to December 2011, the median 
A

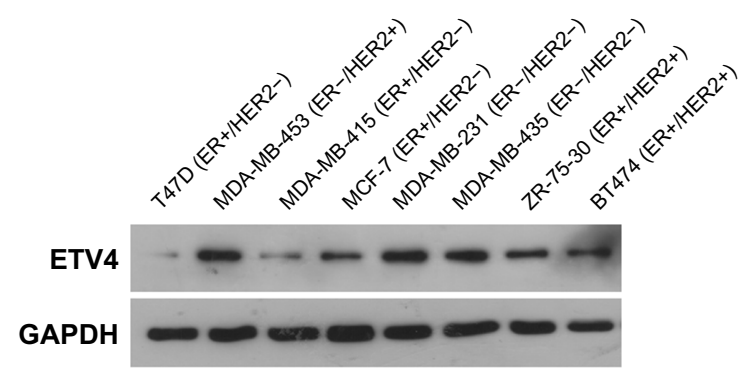

C
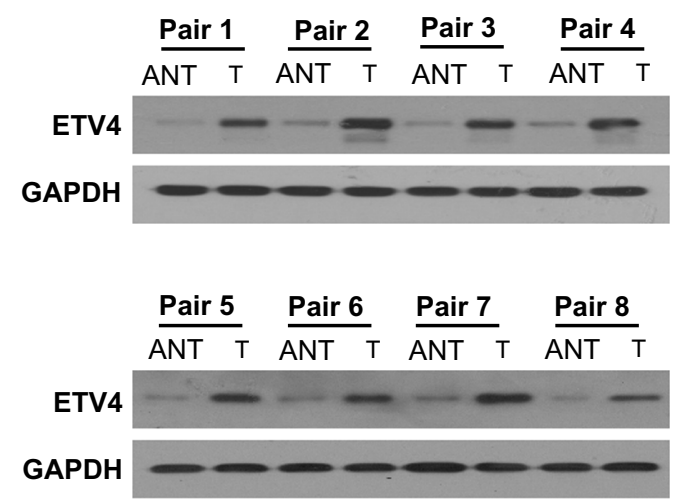

B

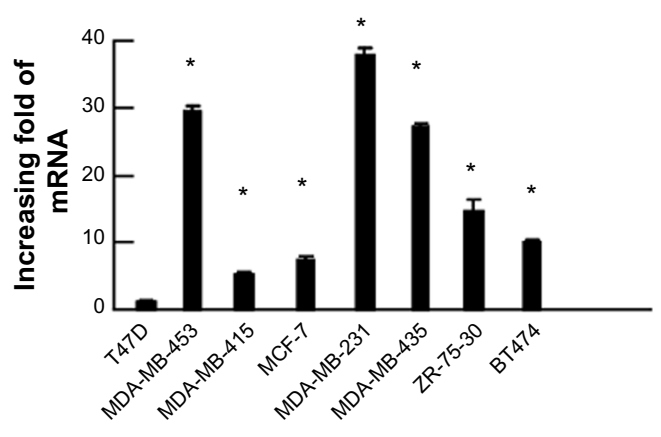

D

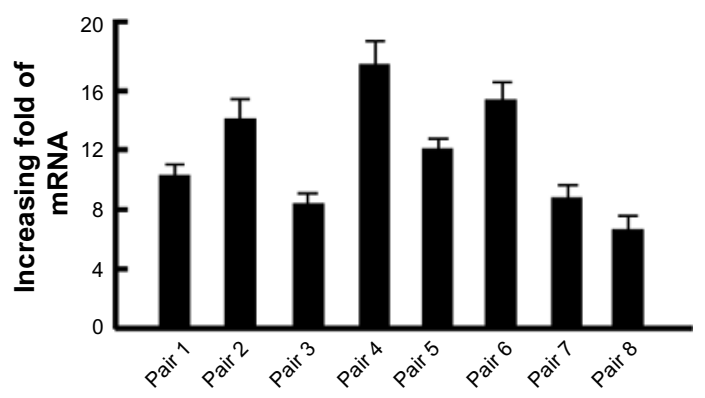

E

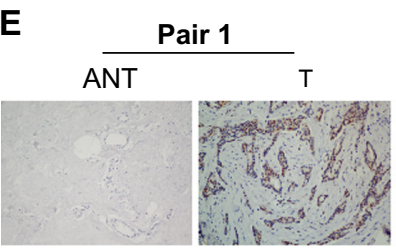

\section{Pair 5}

ANT

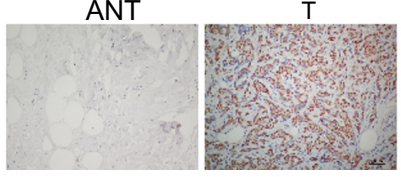

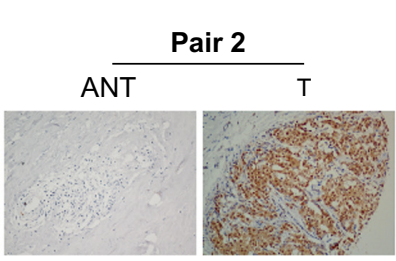

Pair 6 ANT
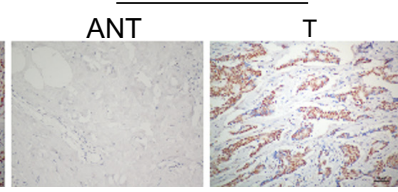
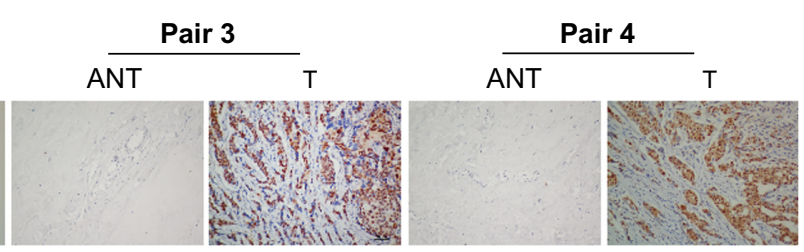

Figure 2 Verification of ETS translocation variant 4 (ETV4) overexpression in triple-negative breast cancer (TNBC) cell lines and tissue.

Notes: (A) Western blot analysis of ETV4 protein expression in TNBC cell lines and hormone receptor-positive cell lines. Glyceraldehyde 3-phosphate dehydrogenase (GAPDH) was used as a loading control. (B) Real-time reverse transcription analysis of ETV4 messenger ribonucleic acid (mRNA) expression in TNBC cell lines and hormone receptor-positive cell lines. Transcript levels were normalized to GAPDH. (C) Western blot analysis of ETV4 protein expression in TNBC tissue (T) and adjacent normal breast tissue (ANT); GAPDH was used as a loading control. (D) Real-time reverse transcription analysis of ETV4 mRNA expression in TNBC tissue and adjacent normal breast tissue; expression data were normalized to GAPDH. Error bars represent the mean \pm standard deviation from three independent experiments; $* P<0.05$. (E) Immunohistochemistry staining of corresponding TNBC tissue (T) and adjacent normal breast tissue (ANT).

Abbreviation: ER, estrogen receptor.

follow-up time was 74 months (range, $8-150$ months). Among all 135 patients, 30 cases relapsed and 28 cases died. The 5-year DFS and OS of all patients were $74.5 \%$ and $85.1 \%$, respectively. According to the sites of first relapse, ten patients had developed a locoregional recurrence and 26 patients had developed a distant metastasis. When the patients were stratified in terms of the expression level of ETV4 protein, the 5-year DFS in tumors with low ETV4 expression and high expression was $89.7 \%$ and $63.4 \%$, respectively $(P=0.001)$. The 5 -year OS of patients with low ETV4 expression and high expression was $93.1 \%$ and $79.2 \%$, respectively $(P=0.017)$. Compared with low ETV4 


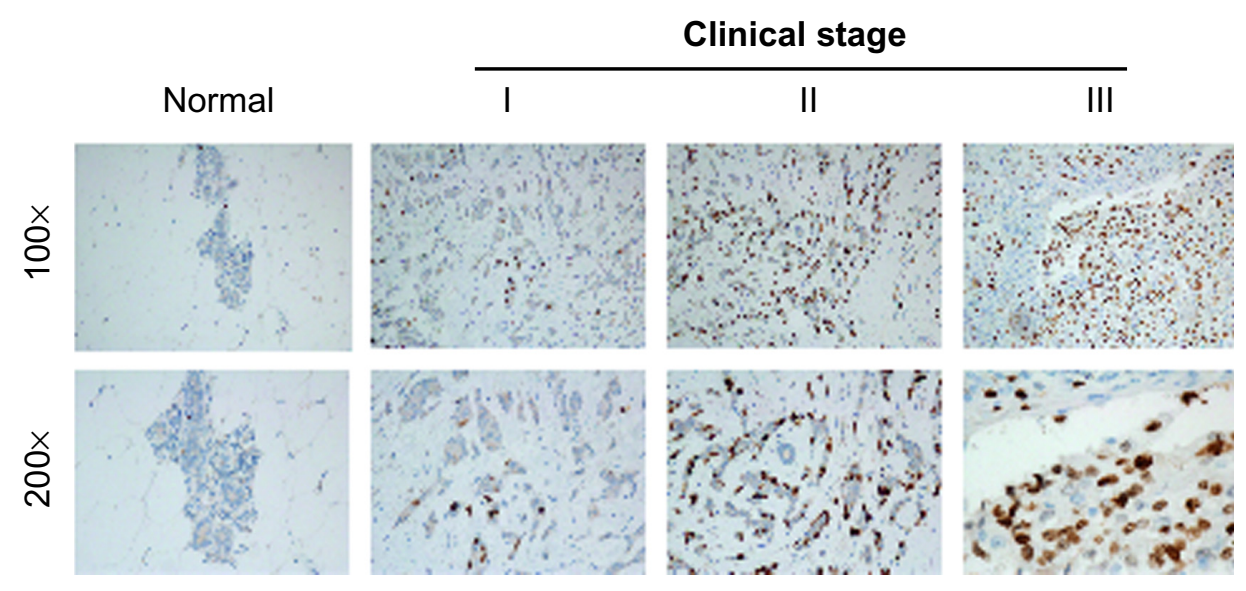

Figure 3 Representative ETS translocation variant 4 immunohistochemistry staining of normal breast tissue and archived triple-negative breast cancer tissue.

protein-expressed tumor, patients with high expression of ETV4 protein had a significantly higher risk in developing a distant metastasis $(97.4 \%$ vs $73.1 \%$, Fisher's exact test, $P<0.0001$ ), whereas the risk for locoregional recurrence was not significantly different between these two groups (96.5\% vs $90.7 \%, P=0.287$ ) (Figure $4 \mathrm{~A}-\mathrm{D}$ ).

Univariate and multivariate analyses were performed and we found that positive lymph nodes, higher clinical stage, present lymphovascular invasion, and expression level of
ETV4 protein were significantly associated with reduced DFS and OS in univariate analysis (Tables 3 and 4). Furthermore, the multivariate analysis showed that only high expression of ETV4 protein remained an independent prognostic factor for shorter DFS $(P=0.021)$ (Table 3).

\section{Discussion}

In this study, we first detected a total of eleven differentially expressed genes in TNBC tissue by human tumor metastasis

Table 2 Association ETV4 protein expression and clinicopathological parameters from TNBC patients

\begin{tabular}{|c|c|c|c|c|}
\hline \multirow[t]{2}{*}{ Variable } & \multirow{2}{*}{$\begin{array}{l}\text { Total } \\
\text { population }\end{array}$} & \multicolumn{2}{|l|}{ ETV4 } & \multirow[t]{2}{*}{$P$-value } \\
\hline & & Underexpression & Overexpression & \\
\hline Total & 135 & $58(43.0)$ & $77(57.0)$ & \\
\hline Median age (range), years & $49(16-76)$ & $49(27-76)$ & $49(16-72)$ & 0.64 I (Mann-Whitney) \\
\hline \multicolumn{5}{|l|}{ Age, n (\%) } \\
\hline$\leq 35$ years & $31(23.0)$ & $15(48.4)$ & $16(51.6)$ & 0.311 \\
\hline$>35$ years & $104(77.0)$ & $43(4 \mid .3)$ & 61 (58.7) & \\
\hline \multicolumn{5}{|l|}{ Menopausal status, n (\%) } \\
\hline Premenopausal & $78(57.8)$ & $34(43.6)$ & $44(56.4)$ & 0.502 \\
\hline Postmenopausal & $57(42.2)$ & $24(42.1)$ & $33(57.9)$ & \\
\hline \multicolumn{5}{|l|}{ Tumor size, n (\%) } \\
\hline$\leq 2 \mathrm{~cm}$ & $33(24.4)$ & $18(54.5)$ & I5 (45.5) & 0.090 \\
\hline$>2 \mathrm{~cm}$ & $102(75.6)$ & $40(39.2)$ & $62(60.8)$ & \\
\hline \multicolumn{5}{|l|}{ Lymph node status, n (\%) } \\
\hline Negative & $63(46.7)$ & $37(58.7)$ & $26(4 \mid .3)$ & $<0.0001$ \\
\hline Positive & $72(53.3)$ & $21(29.2)$ & $5 \mathrm{I}(70.8)$ & \\
\hline \multicolumn{5}{|l|}{ AJCC stage, $n(\%)$} \\
\hline $1 / I I$ & $93(68.9)$ & $52(55.9)$ & $4 I(44.1)$ & $<0.0001$ \\
\hline III & $42(31.1)$ & $6(14.3)$ & $36(85.7)$ & \\
\hline \multicolumn{5}{|l|}{ Histological grade, n (\%) } \\
\hline $\mathrm{I} / \mathrm{II}$ & $39(28.9)$ & $20(51.3)$ & 19 (48.7) & 0.146 \\
\hline III & $96(7 I . I)$ & $38(39.6)$ & $58(60.4)$ & \\
\hline \multicolumn{5}{|c|}{ Lymphovascular invasion, n (\%) } \\
\hline Yes & $36(26.7)$ & $8(22.2)$ & $28(77.8)$ & 0.003 \\
\hline No & $99(73.3)$ & $50(50.5)$ & $49(49.5)$ & \\
\hline
\end{tabular}

Note: The chi-square test was used.

Abbreviations: ETV4, ETS translocation variant 4; TNBC, triple-negative breast cancer; AJCC, American Joint Committee on Cancer. 
A

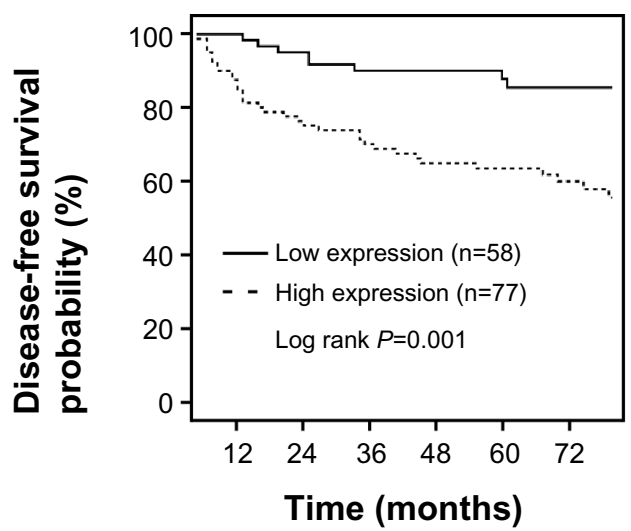

C

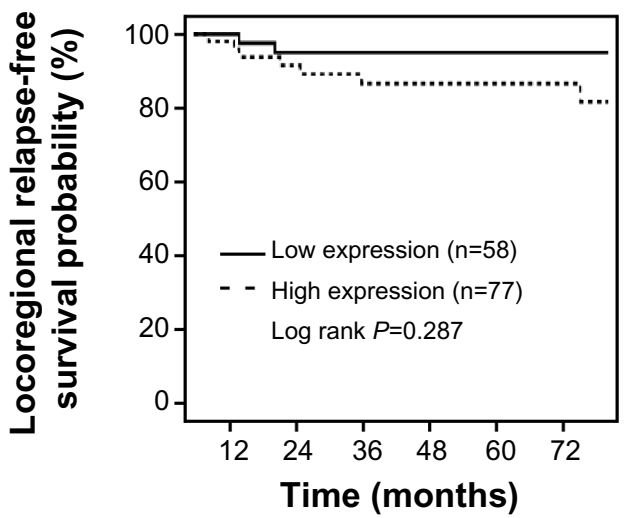

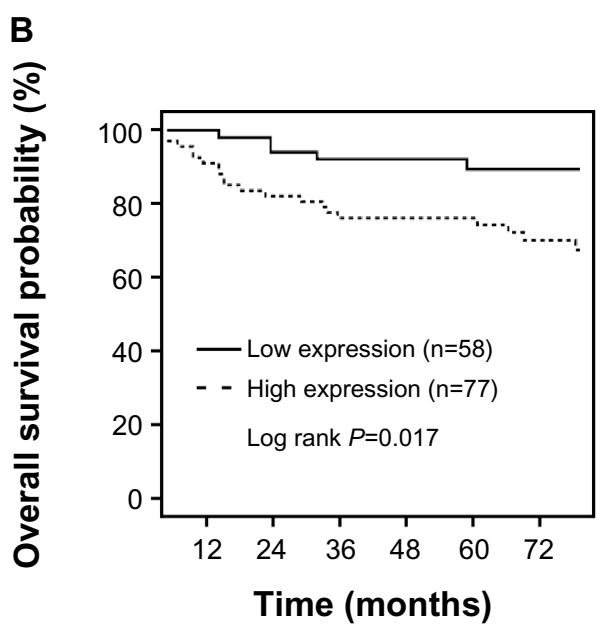

D

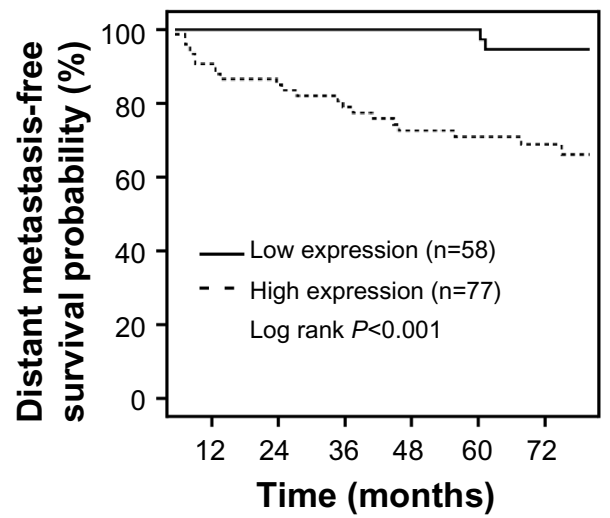

Figure 4 Association of ETS translocation variant 4 expression with poor prognosis of triple-negative breast cancer patients. Notes: (A) Disease-free survival; (B) overall survival; (C) locoregional relapse-free survival; (D) distant metastasis-free survival.

PCR array analysis, of which ETV4 was the most differentially expressed gene $(P=0.0033$, Table 1$)$. In addition, some studies suggested that ETV4 could promote cancer development and metastasis, ${ }^{12-14,16,17}$ so we selected an ETV4 gene for further study. Our findings verified ETV4 overexpression in breast cancer cell lines and tissue samples. ETV4 protein was overexpressed in $57.0 \%$ of TNBC cases. ETV4 overexpression was associated with an advanced stage and a higher proportion of positive lymph node and lymphovascular invasion. Patients with an ETV4-overexpressed tumor had a significantly higher risk of developing distant metastasis and poor OS and DFS. Multivariate analysis showed that overexpression of ETV4 protein was an independent predictor of poor DFS of TNBC patients. The current study demonstrated that overexpression of ETV4 protein had an increased risk for developing distant metastasis and poor survival in TNBC patients. Further study

Table 3 Univariate and multivariate analysis of disease-free survival in I35 patients with triple-negative breast cancer

\begin{tabular}{|c|c|c|c|c|c|c|c|}
\hline \multirow[t]{2}{*}{ Variables } & \multirow[t]{2}{*}{$\mathbf{N}$} & \multicolumn{3}{|c|}{ Univariate } & \multicolumn{3}{|c|}{ Multivariate } \\
\hline & & $\mathbf{H R}^{\mathbf{a}}$ & $95 \% \mathrm{Cl}^{\mathrm{a}}$ & $P$-value & $\mathbf{H R}^{\mathbf{a}}$ & $95 \% \mathrm{Cl}^{\mathrm{a}}$ & P-value \\
\hline Age, years $(\leq 35$ vs $>5)$ & $31 / 104$ & 2.15 & $0.9 I-5.11$ & 0.083 & 1.86 & $0.76-4.51$ & 0.172 \\
\hline Tumor size, $\mathrm{cm}(\leq 2$ vs $>2)$ & $33 / 102$ & 0.89 & $0.45-1.77$ & 0.739 & 0.75 & $0.36-1.58$ & 0.452 \\
\hline Node status (negative vs positive) & $63 / 72$ & 2.89 & $1.45-5.73$ & 0.002 & $\mathrm{I} .84$ & $0.80-4.30$ & 0.160 \\
\hline Stage (I/II vs III) & $93 / 42$ & 2.18 & $1.20-3.98$ & 0.011 & 0.98 & $0.34-2.86$ & 0.977 \\
\hline Histological grade (I/II vs III) & $39 / 96$ & 1.17 & $0.59-2.31$ & 0.662 & 0.94 & $0.46-1.94$ & 0.875 \\
\hline Lymphovascular invasion (no vs yes) & $99 / 36$ & 2.10 & $1.14-3.88$ & 0.018 & $\mathrm{I} .33$ & $0.45-3.94$ & 0.602 \\
\hline ETV4 expression (lower vs high ) & $58 / 77$ & 3.27 & $1.57-6.83$ & 0.002 & 2.58 & I. I5-5.77 & 0.021 \\
\hline
\end{tabular}

Note: ${ }^{a} \mathrm{HR}$ and $95 \% \mathrm{Cls}$ were calculated using Cox regression analysis.

Abbreviations: $\mathrm{HR}$, hazard ratio; $\mathrm{Cl}$, confidence interval; ETV4, ETS translocation variant 4. 
Table 4 Univariate and multivariate analysis of overall survival in I35 patients with triple-negative breast cancer

\begin{tabular}{|c|c|c|c|c|c|c|c|}
\hline \multirow[t]{2}{*}{ Variables } & \multirow[t]{2}{*}{$\mathbf{N}$} & \multicolumn{3}{|c|}{ Univariate } & \multicolumn{3}{|c|}{ Multivariate } \\
\hline & & $\mathbf{H R}^{\mathbf{a}}$ & $95 \% \mathrm{Cl}^{\mathrm{a}}$ & $P$-value & $\mathbf{H R}^{\mathbf{a}}$ & $95 \% \mathrm{Cl}^{\mathrm{a}}$ & P-value \\
\hline Age, years $(\leq 35$ vs $>35)$ & $31 / 104$ & 2.96 & $0.89-9.82$ & 0.077 & 2.81 & $0.82-9.61$ & 0.099 \\
\hline Tumor size, $\mathrm{cm}(\leq 2$ vs $>2$ ) & $33 / 102$ & 0.85 & $0.36-2.02$ & 0.711 & 0.64 & $0.25-1.63$ & 0.349 \\
\hline Node status (negative vs positive) & $63 / 72$ & 2.77 & $|| 8-6.5 \mid$. & 0.020 & 1.45 & $0.49-4.30$ & 0.505 \\
\hline Stage (I/II vs III) & $93 / 42$ & 2.34 & $1.11-4.9 \mid$ & 0.025 & 0.81 & $0.18-3.62$ & 0.786 \\
\hline Histological grade (I/II vs III) & $39 / 96$ & 1.06 & $0.46-2.41$ & 0.895 & 1.04 & $0.44-2.46$ & 0.925 \\
\hline Lymphovascular invasion (no vs yes) & $99 / 36$ & 2.56 & $1.22-5.39$ & 0.013 & 2.26 & $0.51-9.93$ & 0.280 \\
\hline ETV4 expression (lower vs high) & $58 / 77$ & 2.85 & I.16-7.04 & 0.023 & 2.09 & $0.76-5.76$ & 0.153 \\
\hline
\end{tabular}

Note: a ${ }^{\mathrm{HR}}$ and $95 \% \mathrm{Cls}$ were calculated using Cox regression analysis.

Abbreviations: $\mathrm{HR}$, hazard ratio; $\mathrm{Cl}$, confidence interval; ETV4, ETS translocation variant 4.

will investigate whether ETV4 protein is a novel target for control of TNBC distant metastasis.

TNBC is characterized by a high probability of a distant metastasis, especially to the lung and brain, ${ }^{4-6}$ which is due to aberrant expression of tumor metastasis-related genes. ${ }^{7-9}$ Thus, in this study, we profiled differentially expression of some of these genes and found that a total of eleven such genes were altered. We then chose ETV4 for further verification and analysis. Indeed, ETV4 belongs to the PEA3 group of the Ets family of transcription factors, expression of which was suggested to be associated with tumor metastases for several types of human cancers. ${ }^{13,23-27}$ In this study, we confirmed that ETV4 was one of the most significantly upregulated genes in TNBC tissue.

Furthermore, in the current study, we showed that expression of both ETV4 mRNA and protein was noticeably upregulated in ER-negative cell lines, regardless of HER2 status. Our current data were in agreement with those reported recently by Baert et al. ${ }^{18}$ We also showed that expression of both ETV4 mRNA and protein in TNBC tissue was significantly higher than in the adjacent normal breast tissue. With further examination by IHC in our TMAs of TNBC, we found that $57.0 \%$ of TNBC tissue exhibited high expression of ETV4 protein. A similar frequency (53\%) of ETV4 protein expression was observed by Qin et al. ${ }^{28}$ Nevertheless, Bieche et al ${ }^{19}$ reported that expression of ETV4 mRNA was detected in only $14 \%$ of unselected breast cancer tissue, and the level of ETV4 expression had a significant negative correlation with the level of ER, while Benz et al ${ }^{29}$ reported that ETV4 mRNA expression was overexpressed in $76 \%$ of Her2-positive breast cancer tissue. It is unknown what contributed to this discrepancy. However, it is well known that both TNBC and Her2-positive breast cancer represent two aggressive breast cancer subtypes and they share several common characteristics such as a higher risk of distant metastasis and a poor prognosis. ${ }^{4,530}$ These findings suggested that ETV4 might be especially highly expressed in breast cancer cells with an invasive phenotype.
In addition, in this study, we found that overexpression of ETV4 protein was positively associated with lymph node involvement, lymphovascular invasion, and advanced clinical stage. Patients with ETV4 overexpression had a significantly shorter DFS and OS. Importantly, our results also show that overexpression of ETV4 protein increased the risk of developing a distant metastasis in TNBC patients, rather than locoregional recurrence. These data further suggest that ETV4 overexpression could promote progression and metastasis of TNBC. Our further multivariate analysis showed that ETV4 overexpression was an independent predictor of short DFS of TNBC patients. This result is consistent with previous studies in other cancers. . $^{25,27,31,32}$

Mechanistically, the PEA3 subfamily of ETS-domain transcription factors has been shown to be an important driver of cancer cell metastasis. ${ }^{23}$ Overexpressed ETV4 enhances transcription of different target genes involved in tumor metastasis, such as MMP genes. ${ }^{26,27,33}$ These latter genes are involved in degrading the extracellular matrix to permit cancer metastasis. ${ }^{34-36}$

\section{Conclusion}

Our current study detected eleven differentially expressed tumor metastasis-related genes in TNBC tissue, and further investigation showed that ETV4 upregulated in more than half of the TNBC tissue specimens. ETV4 overexpression was not only a marker of tumor aggressiveness but also a poor prognostic marker for ER-negative patients, including TNBC. Further studies of ETV4 could help to define the molecular mechanism responsible for TNBC distant metastasis and offer potentially novel strategies for future control of TNBC distant metastasis.

\section{Acknowledgments}

This study was financially supported by the Science and Technology Program of Guangdong Province (No 2010B031600219) and by the National Natural 
Science Foundation of China (Grant No 81372820). The funders had no role in study design, data collection and analysis, decision to publish, or preparation of the manuscript.

\section{Disclosure}

The authors report no conflicts of interest in this work.

\section{References}

1. Onitilo AA, Engel JM, Greenlee RT, et al. Breast cancer subtypes based on ER/PR and Her2 expression: comparison of clinicopathologic features and survival. Clin Med Res. 2009;7:4-13.

2. Bauer KR, Brown M, Cress RD, et al. Descriptive analysis of estrogen receptor (ER)-negative, progesterone receptor (PR)-negative, and HER2-negative invasive breast cancer, the so-called triple-negative phenotype: a population-based study from the California Cancer Registry. Cancer. 2007;109:1721-1728.

3. Rakha EA, El-Sayed ME, Green AR, et al. Prognostic markers in triplenegative breast cancer. Cancer. 2007;109:25-32.

4. Lin NU, Claus E, Sohl J, et al. Sites of distant recurrence and clinical outcomes in patients with metastatic triple-negative breast cancer: high incidence of central nervous system metastases. Cancer. 2008;113:2638-2645.

5. Hines SL, Vallow LA, Tan WW, et al. Clinical outcomes after a diagnosis of brain metastases in patients with estrogen- and/or human epidermal growth factor receptor 2-positive versus triple-negative breast cancer. Ann Oncol. 2008;19:1561-1565.

6. Dent R, Trudeau M, Pritchard KI, et al. Triple-negative breast cancer: clinical features and patterns of recurrence. Clin Cancer Res. 2007;13:4429-4434.

7. Kang Y, Siegel PM, Shu W, et al. A multigenic program mediating breast cancer metastasis to bone. Cancer Cell. 2003;3:537-549.

8. Minn AJ, Gupta GP, Siegel PM, et al. Genes that mediate breast cancer metastasis to lung. Nature. 2005;436:518-524.

9. Minn AJ, Kang Y, Serganova I, et al. Distinct organ-specific metastatic potential of individual breast cancer cells and primary tumors. $J$ Clin Invest. 2005;115:44-55.

10. Keld R, Guo B, Downey P, et al. PEA3/ETV4-related transcription factors coupled with active ERK signalling are associated with poor prognosis in gastric adenocarcinoma. $\mathrm{Br} J$ Cancer. 2011;105:124-130

11. Oh S, Shin S, Janknecht R. ETV1, 4 and 5: An oncogenic subfamily of ETS transcription factors. Biochim Biophys Acta. 2012;1826:1-12.

12. de Launoit $Y$, Chotteau-Lelievre A, Beaudoin C, et al. The PEA3 group of ETS-related transcription factors. Role in breast cancer metastasis. Adv Exp Med Biol. 2000;480:107-116.

13. Horiuchi S, Yamamoto H, Min Y, et al. Association of ets-related transcriptional factor E1 AF expression with tumour progression and overexpression of MMP-1 and matrilysin in human colorectal cancer. J Pathol. 2003;200:568-576.

14. Cowden Dahl KD, Zeineldin R, Hudson LG. PEA3 is necessary for optimal epidermal growth factor receptor-stimulated matrix metalloproteinase expression and invasion of ovarian tumor cells. Mol Cancer Res. 2007;5:413-421.

15. Keld R, Guo B, Downey P, et al. The ERK MAP kinase-PEA3/ETV4MMP-1 axis is operative in oesophageal adenocarcinoma. Mol Cancer. 2010;9:313.

16. Shepherd TG, Kockeritz L, Szrajber MR, et al. The pea3 subfamily ets genes are required for HER2/Neu-mediated mammary oncogenesis. Curr Biol. 2001;11:1739-1748.
17. Kapila S, Xie Y, Wang W. Induction of MMP-1 (collagenase-1) by relaxin in fibrocartilaginous cells requires both the AP-1 and PEA-3 promoter sites. Orthod Craniofac Res. 2009;12:178-186.

18. Baert JL, Monte D, Musgrove EA, et al. Expression of the PEA3 group of ETS-related transcription factors in human breast-cancer cells. Int $J$ Cancer. 1997;70:590-597.

19. Bieche I, Tozlu S, Girault I, et al. Expression of PEA3/E1AF/ETV4, an Ets-related transcription factor, in breast tumors: positive links to MMP2, NRG1 and CGB expression. Carcinogenesis. 2004;25:405-411.

20. Davidson B, Goldberg I, Tell L, et al. The clinical role of the PEA3 transcription factor in ovarian and breast carcinoma in effusions. Clin Exp Metastasis. 2004;21:191-199.

21. Xia WY, Lien HC, Wang SC, et al. Expression of PEA3 and lack of correlation between PEA3 and HER-2/neu expression in breast cancer. Breast Cancer Res Treat. 2006;98:295-301.

22. He J, Peng R, Yuan Z, et al. Prognostic value of androgen receptor expression in operable triple-negative breast cancer: a retrospective analysis based on a tissue microarray. Med Oncol. 2012;29:406-410.

23. de Launoit Y, Baert JL, Chotteau-Lelievre A, et al. The Ets transcription factors of the PEA3 group: transcriptional regulators in metastasis. Biochim Biophys Acta. 2006;1766:79-87.

24. Zhu X, Jiang J, Shen H, et al. Elevated beta1,4-galactosyltransferase I in highly metastatic human lung cancer cells. Identification of E1AF as important transcription activator. J Biol Chem. 2005;280: $12503-12516$

25. Yamamoto H, Horiuchi S, Adachi Y, et al. Expression of ets-related transcriptional factor E1AF is associated with tumor progression and over-expression of matrilysin in human gastric cancer. Carcinogenesis. 2004;25:325-332.

26. Shindoh M, Higashino F, Kaya M, et al. Correlated expression of matrix metalloproteinases and ets family transcription factor E1A-F in invasive oral squamous-cell-carcinoma-derived cell lines. Am J Pathol. 1996;148:693-700.

27. Hida K, Shindoh M, Yoshida K, et al. Expression of E1AF, an ets-family transcription factor, is correlated with the invasive phenotype of oral squamous cell carcinoma. Oral Oncol. 1997;33:426-430.

28. Qin L, Liao L, Redmond A, et al. The AIB1 oncogene promotes breast cancer metastasis by activation of PEA3-mediated matrix metalloproteinase 2 (MMP2) and MMP9 expression. Mol Cell Biol. 2008;28:5937-5950.

29. Benz CC, O'Hagan RC, Richter B, et al. HER2/Neu and the Ets transcription activator PEA3 are coordinately upregulated in human breast cancer. Oncogene. 1997;15:1513-1525.

30. Ross JS, Slodkowska EA, Symmans WF, et al. The HER-2 receptor and breast cancer: ten years of targeted anti-HER-2 therapy and personalized medicine. Oncologist. 2009;14:320-368.

31. Myers E, Hill AD, Kelly G, et al. A positive role for PEA3 in HER2-mediated breast tumour progression. Br J Cancer. 2006;95:1404-1409.

32. Davidson B, Goldberg I, Gotlieb WH, et al. PEA3 is the second Ets family transcription factor involved in tumor progression in ovarian carcinoma. Clin Cancer Res. 2003;9:1412-1419.

33. Habelhah H, Okada F, Kobayashi M, et al. Increased E1AF expression in mouse fibrosarcoma promotes metastasis through induction of MT1-MMP expression. Oncogene. 1999;18:1771-1776.

34. Duffy MJ, Maguire TM, Hill A, et al. Metalloproteinases: role in breast carcinogenesis, invasion and metastasis. Breast Cancer Res. 2000;2:252-257.

35. Oikawa T. ETS transcription factors: possible targets for cancer therapy. Cancer Sci. 2004;95:626-633.

36. Higashino F, Yoshida K, Noumi T, et al. Ets-related protein E1A-F can activate three different matrix metalloproteinase gene promoters Oncogene. 1995;10:1461-1463. 


\section{Publish your work in this journal}

OncoTargets and Therapy is an international, peer-reviewed, open access journal focusing on the pathological basis of all cancers, potential targets for therapy and treatment protocols employed to improve the management of cancer patients. The journal also focuses on the impact of management programs and new therapeutic agents and protocols on

patient perspectives such as quality of life, adherence and satisfaction. The manuscript management system is completely online and includes a very quick and fair peer-review system, which is all easy to use. Visit http://www.dovepress.com/testimonials.php to read real quotes from published authors.

Submit your manuscript here: http://www.dovepress.com/oncotargets-and-therapy-journal 